\title{
JAK/STAT signaling regulates tissue outgrowth and male germline stem cell fate in Drosophila
}

\author{
Shree Ram SINGH, Xiu CHEN, Steven X. HOU*
}

The Laboratory of Immunobiology, National Institutes of Health, National Cancer Institute at Frederick, Frederick, MD 21702, USA

\begin{abstract}
In multicellular organisms, biological activities are regulated by cell signaling. The various signal transduction pathways regulate cell fate, proliferation, migration, and polarity. Miscoordination of the communicative signals will lead to disasters like cancer and other fatal diseases. The JAK/STAT signal transduction pathway is one of the pathways, which was first identified in vertebrates and is highly conserved throughout evolution. Studying the JAK/STAT signal transduction pathway in Drosophila provides an excellent opportunity to understand the molecular mechanism of the cell regulation during development and tumor formation. In this review, we discuss the general overview of JAK/STAT signaling in Drosophila with respect to its functions in the eye development and stem cell fate determination.
\end{abstract}

Keywords: cell signaling, JAK/STAT signal transduction pathway, cell regulation, Drosophila, stem cell fate, tissue outgrowth.

\section{INTRODUCTION}

The Janus kinase (JAK)/signal transducer and activator of transcription (STAT) cascade is a signal transduction pathway that was originally identified in the vertebrate system $[1,2]$ and has been extensively studied during the last decade in several model organisms, including Drosophila $[1,3,4,5]$. This pathway was identified through studies on the transcriptional activation response to a variety of cytokines and growth factors [6,7] and is highly conserved and capable of transmitting a multitude of signals for development and homeostasis in animals from flies to humans.

In the mammalian system, the JAK/STAT pathway plays a central role in transducing the cytokine signal and regulates various biological processes such as cell growth, differentiation, migration, apoptosis, fetal development, transformation, inflammation, and immune response. The constitutive activation of JAK/STAT is correlated with several oncogenic transformations, such as leukemia, lymphoma, and multiple myeloma, as well as some solid neoplasias, head, neck, brain, breast, lung, pancreas, prostate, and ovarian cancers $[8,9]$.

*Correspondence: Steven X. HOU

Tel: 301-846-1589; fax: 301-846-6145;

E-mail: shou@mail.ncifcrf.gov

\section{JAK/STAT SIGNALING IN DROSOPHILA}

The JAK/STAT pathway is also present in the Drosophila genetic model system $[1,4,10]$. The Drosophila JAK/STAT pathway has four main components: JAK, encoded by the hopscotch (hop) gene [11]; STAT, encoded by the stat $92 E$ gene $[12,13]$; a ligand, encoded by the unpaired (upd) gene [14]; a receptor, encoded by the domeless (dome [15]) /master of marelle (mom [16]) gene. The current model of the Hop/Stat92E signal cascade is the following: The extracellular Upd protein binds and activates the receptor Dome/Mom, which in turn activates the intracellular Hop/JAK; Hop/JAK then phosphorylates and stabilizes Stat92E. The phosphorylated Stat92E dimerizes and translocates to the nucleus to activate the gene transcription [1, 3, 17, 18] (Fig. 1).

The JAK/STAT signal transduction pathway regulates various developmental processes in Drosophila, including embryonic segmentation, sex determination, eye formation, tracheal formation, male germline stem cell self-renewal, border cell migration, polar and follicular cell fate determination in the female germline, germ cell proliferation and invasive migration $[1,3,17,19,20,21]$. Furthermore, hyperactivation of this pathway in Drosophila leads to melanotic- or leukemia-like tumor formation. In this review, we will focus on the pathway's functions in the eye development and male germline stem cell fate determination. 


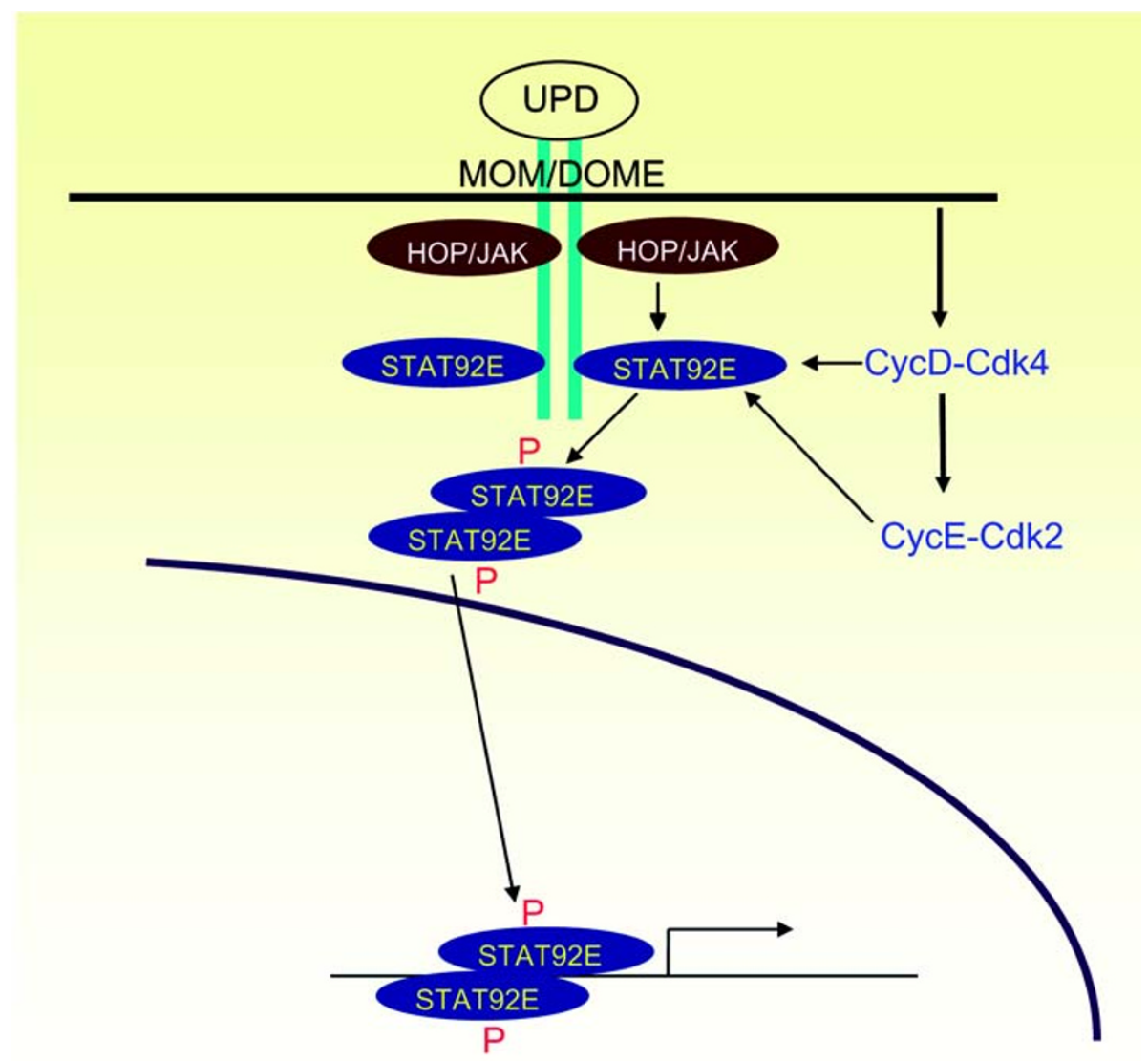

Fig. 1 The Drosophila JAK/STAT pathway.

\section{Role in cell proliferation during eye development}

In both mammalian and Drosophila systems, the JAK/ STAT signaling pathway plays a crucial role in controlling organ or tissue size. The Drosophila eye is a complex neural tissue with precise cellular architecture and composed of approximately 800 subunits called ommatidia, each of which contains eight photoreceptors and 12 accessory cells arranged in an invariant pattern [22]. The role of the JAK/ STAT pathway in the eye development of Drosophila has been studied genetically $[10,18]$. The activity of upd and hop is required for the proliferation and/or survival of eye imaginal cells $[10,16]$. Strong alleles of upd are embryonic lethal, and weaker alleles such as $o s^{l}$ and $o s^{s}$ give rise to adult flies that have small eyes [14]. Loss of hop activity results in the absence of proliferating diploid imaginal cells throughout the larva, and some transheterozygous combinations of alleles give rise to adults that have a small eye or eyeless phenotype [10,23]. Overexpression of dpias (the negative regulator of Stat92E) results in small and rough eyes [24]. Further, the $o s^{l}$ small eye phenotype could be partially suppressed by reducing the dpias gene dosage.
Genetic interaction experiments suggest that the correct dpias/stat $92 E$ ratio is crucial for eye imaginal cell growth and differentiation [24]. Upd is expressed in the center of the posterior margin of the eye disc [25]. Loss-of-function upd mutations result in a small eye size, and ectopic misexpression of upd causes enlargement of the eye [16, 24, 25]. upd regulates eye size through the Mom/Hop/ Stat92E signaling pathway to promote cell proliferation, by accelerating the cell cycle in the undifferentiated cells from anterior to the MF (morphogenetic furrow).

In the eye, excess Hop/Stat92E signaling induces cell overproliferation, and excess $\mathrm{CycD}-\mathrm{Cdk} 4$ activity blocks differentiation and induces overgrowth. Our group recently demonstrated that excess Hop/Stat92E signaling can synergize with both CycD-Cdk4 and CycE-Cdk2 in melanotic tumors, but specifically synergizes with CycD-Cdk4, not $\mathrm{CycE}-\mathrm{Cdk} 2$, to promote the formation of an enlarged eye with extra ommatidia [19]. These results demonstrate that the JAK/STAT pathway and Cyclin-Cdk cooperatively regulate tissue outgrowth and tumor formation in Drosophila. 


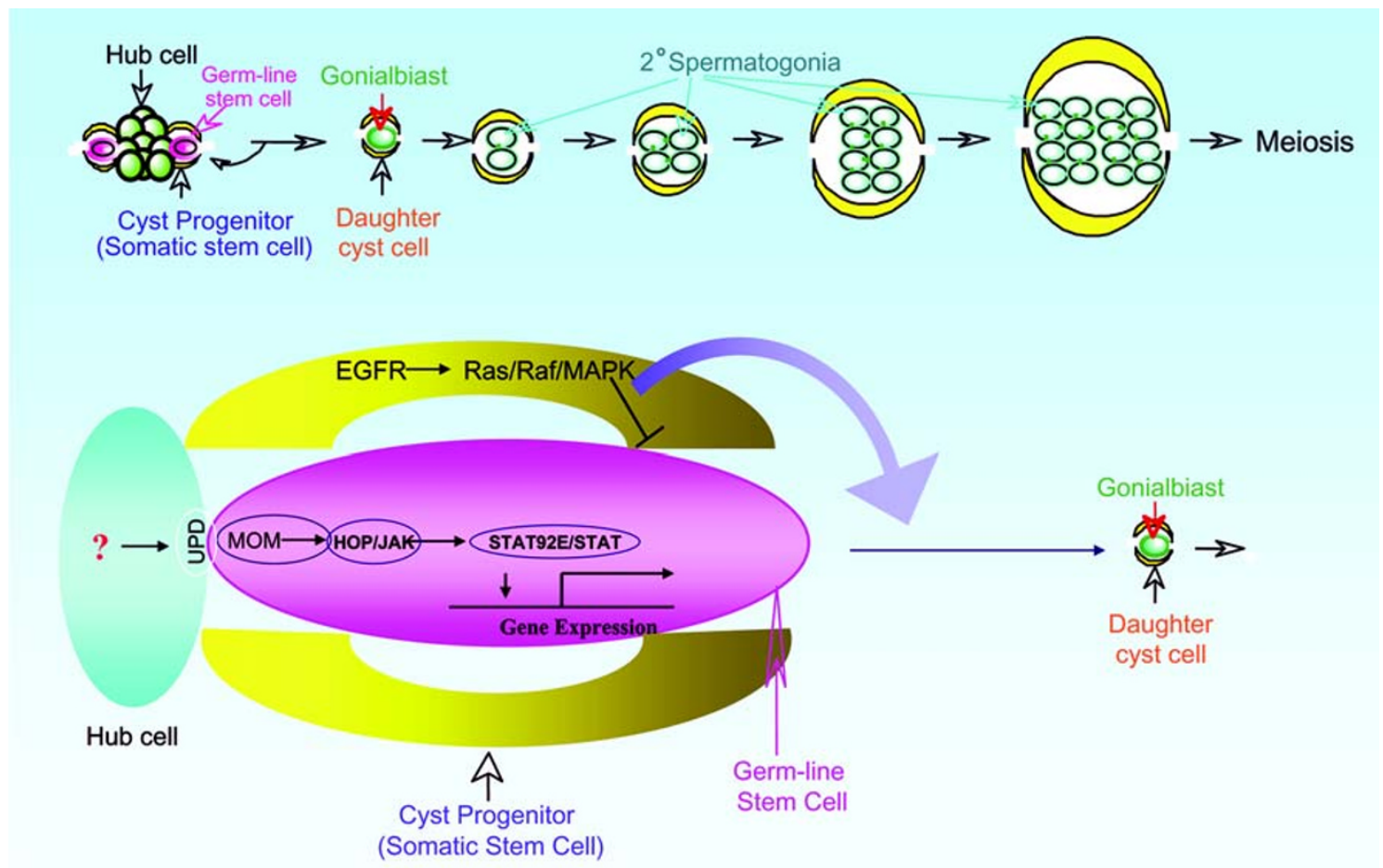

Fig. 2 The HOP/STAT92E pathway regulates male germline stem cell self-renewal and differentiation. (A) Five to nine germline stem cells (only two GSCs are shown in this scheme) are surrounded by approximately twice as many cyst progenitor cells (somatic stem cell), and both are anchored around somatic hub cells at the tip of the Drosophila testis. The testis proliferation center is composed of hub, germline, and somatic stem cells, gonialblasts, and $2^{\circ}$ spermatogonia. (B) Schematic diagram showing the cooperative regulation of the male germline stem cell self-renewal and differentiation by HOP/STAT92E and RAS/MAPK pathways.

Although it remains to be seen whether cooperation of CycD-Cdk 4 and the JAK/STAT pathway in regulating tissue growth is a general phenomena in all species, abundant evidence shows that CycD-Cdk4 and the JAK/STAT pathway regulate similar biological processes in both flies [1] and mammals [2, 26]. Just as overexpressing CycDCdk4 in the fly wing or eye causes hyperplasia, targeted overexpression of CycD1 in mice can promote epidermal, mammary, and thymic hyperplasia. These parallels are also evident in loss-of-function studies. Both CycD1 and Cdk4 knock-out mice are smaller than their littermates and exhibit decreased growth rates. Cdk4 mutant flies are also small. Both male and female Stat5a/b-deficient mice are small with reduced size of fat pads and reduced levels of insulin-like growth factor-1. As discussed above, reduction of the Hop/STAT92E signaling gives rise to adult flies that have a small eye or eyeless phenotype. Taken together, all of these observations suggest that both CycD-Cdk4 and the JAK/STAT pathway regulate tissue growth. Further, in the fly, two dominant temperature-sensitive mutations that hyperactivate Hop, $h o p^{T u m-l}$ and $h o p^{T 42}$ lead to melanotic- or leukemia-like tumor formation. Germline mutations in human $\mathrm{Cdk} 4$, a result of the $\mathrm{Arg} 24 \mathrm{Cys}$ substitution, have been identified in familial melanoma [27, 28]. These mutations abrogate the inhibition of Cdk4 activity by its inhibitor, $\mathrm{P} 16^{\mathrm{INK} 4 \mathrm{a}}[27,28]$. Mice with R24C mutation display an increased weight of $5 \%-10 \%$, compared with control littermates. However, $C d k 4^{R 24 C / R 24 C}$ mice did not develop melanoma similar to that observed in humans carrying the same $\mathrm{R} 24 \mathrm{C}$ mutation in Cdk4 [29]. It is likely that predisposition to melanoma requires a second cooperative signal. The JAK/STAT signaling may identify the second signal. The collaboration of upd and $C y c D-C d k 4$ in the fly eye may somehow mimic signal cooperation during tumor formation. $\mathrm{CycD}-\mathrm{Cdk} 4$ promotes cell growth and blocks cell differentiation; likewise, $u p d$ stimulates cell proliferation. Cooperation of the two signals results in a dramatically outgrown tumor-like eye.

More than $80 \%$ of cancers have detectable lesions in one component of the Cdk4 complex (Cdk4, INK4a, D1, and RB) [30]. Many growth factors and components of signal transduction are oncogenes. Our recent results show that CycD-Cdk4 and the Hop/Stat92E pathway collaboratively induce tissue overgrowth and melanotic tumor formation. We suggest that this relationship between coordinated STAT and Cyclin-Cdk signaling could regu- 
late cell fate, the cell cycle, and/or tumor progression in mammals as well. Thus, the powerful genetic manipulations available in Drosophila may make this an ideal system to study cancer.

\section{Role in male germline stem cell}

In last few years, several findings demonstrated that the canonical JAK/STAT pathway in the fly testis regulates germline stem cell self-renewal, maintenance, and differentiation [31, 32, 33]. Spermatogenesis in Drosophila takes place within the tubular testis [34], and at the tip of the Drosophila testis is a germinal proliferation center composed of a cluster of twelve nonmitotic somatic cells called the hub, and a small number of germline stem cells (GSCs; 16-18 in larvae, 5-9 in adult; Fig. 2A). It is reported that stem cells reside in niches, which actually determine their capacity to self-renew or differentiate [35]. An asymmetric or stereotypically oriented division of stem cells controls spermatogenesis in Drosophila, whereby one daughter cell remains at the hub and retains stem cell identity, while the other one is displaced and becomes a founder gonial cell (or gonialblast) that initiates differentiation [36]. These GSCs are also flanked by somatic stem cells (SSCs) known as cyst progenitor cells, which maintain contact with the hub and divide to produce cyst cells that enclose each gonialblast. The gonialblast divides further to produce interconnected spermatogonia, while SSCs grow without further division and enclose the spermatogonial clusters.

The ligand Upd, which is exclusively expressed in the hub cells, maintains the male germline stem cell self-renewal by activation of the JAK/STAT pathway in GSCs (Fig. 2B). Upd normally instructs GSCs to undergo selfrenewal through the Mom/Hop/Stat92E pathway; Stat92E then enters the nucleus to activate expression of genes that instruct the self-renewal of GSCs and SSCs.

Overexpression of the Hop/Stat92E pathway through overexpressing upd leads to unrestricted stem cell division, and loss-of-function mutations in the Hop/Stat92E pathway lead to loss of GSCs, suggesting that signaling maintains stem cell fate or viability $[31,32]$. Furthermore, GSCs null for stat $92 E$ can produce differentiating daughter cells but cannot maintain stem cell fate. These data suggest that Hop/Stat92E signaling instructs stem cell fate rather than maintaining cell viability [31]. Perhaps cells displaced from the hub do not receive sufficiently high levels of Hop/Stat92E signaling to activate specific gene expression and therefore lose self-renewing capacity. Very recently, Brawley and Matunis [33] found that early spermatogonia with limited mitotic divisions can repopulate the niche and can be reverted to stem cell identity by conditionally manipulating the Hop/Stat92E signaling. Further, their results provide a mechanism for the replacement of lost stem cells in an intact stem cell niche and also aid in better understanding the mechanism of tissue regeneration.

In addition to the JAK/STAT signal transduction pathway, the epidermal growth factor receptor (EGFR) / MAP kinase (MAPK) pathway also regulates germline stem cell fates in the fly testis $[37,38]$ (Fig. 2B). The Drosophila EGFR (DER) through Raf/MAPK pathway functions in SSCs. The DER/Raf/MAPK pathway mediates an SSC to GSC signal that restricts self-renewal and promotes differentiation of the GSCs. If the cyst cells do not send the signal to GSCs through DER/Raf/MAPK pathway, both daughters of the GSCs will retain stem cell identity. Loss of function of der or raf in SSCs will break the balance of self-renewal/differentiation and result in an excess number of GSCs and gonialblasts at the expense of differentiated cell types. These data suggest that SSCs play a guardian role to ensure the balance between the self-renewal and differentiation of GSCs [37, 38] (Fig. 2B). The Drosophila testis system is an intriguing parallel to mouse embryonic stem (ES) cells [39], in which the JAK/STAT signaling is required for the maintenance of the ES cells, while the MAP kinase pathway promotes the ES cell differentiation [40]. In both systems, the JAK/STAT signal is counterbalanced by the Ras/Raf/MAPK signal. The two signals may converge on some downstream targets to regulate cell fate determination (Fig. 2B).

\section{REFERENCES}

1 Hou SX, Zheng Z, Chen X, Perrimon N. The Jak/STAT pathway in model organisms: emerging roles in cell movement. Dev Cell 2002; 3:765-78.

2 O'Shea JJ, Gadina M, Schreiber RD. Cytokine signaling in 2002: new surprises in the JAK/STAT pathway. Cell 2002; 109:S12131.

3 Hou XS, Perrimon N. The JAK-STAT pathway in Drosophila. Trends Genet 1997; 13:105-10.

4 Zeidler MP, Bach EA, Perrimon N. The roles of the Drosophila JAK/STAT pathway. Oncogene 2000; 19:2598-606.

5 Luo H, Dearolf CR. The JAK/STAT pathway and Drosophila development. Bioessays. 2001; 23:1138-47.

6 Decker T. Introduction: STATs as essential intracellular mediators of cytokine responses. Cell Mol Life Sci 1999; 55:1505-8.

7 Imada K, Leonard WJ. The JAK-STAT pathway. Mol Immunol 2000; 37:1-11.

8 Bromberg JF, Wrzeszczynska MH, Devgan G, et al. STAT3 as an oncogene. Cell 1999; 98:295-303.

9 Rabinow L. The proliferation of Drosophila in cancer research: a system for the functional characterization of tumor suppressors and oncogenes. Cancer Invest 2002; 20:531-56.

10 Luo H, Asha H, Kockel L, et al. The Drosophila JAK kinase Hopscotch is required for multiple developmental processes in the eye. Dev Biol 1999; 213:432-41.

11 Binari R, Perrimon N. Stripe-specific regulation of pair-rule genes by hopscotch, a putative Jak family tyrosine kinase in Drosophila. Genes Dev 1994; 8:300-12. 
12 Hou SX, Melnick MB, Perrimon N. marelle acts downstream of the Drosophila HOP/JAK kinase and encodes a protein similar to the mammalian STATs. Cell 1996; 84:411-9.

13 Yan R, Small S, Desplan C, Dearolf CR, Darnell JJ. Identification of a Stat gene that functions in Drosophila development. Cell 1996; 84:421-30.

14 Harrison DA, McCoon PE, Binari R, Gilman M, Perrimon N. Drosophila unpaired encodes a secreted protein that activates the JAK signaling pathway. Genes Dev 1998; 12:3252-63.

15 Brown S, Hu N, Castelli-Gair Hombría J. Identification of the first invertebrate interleukin JAK/STAT receptor, the Drosophila gene domeless. Curr Biol 2001;11:1700-5.

16 Chen HW, Chen X, Oh SW, et al. mom identifies a receptor for the Drosophila JAK/STAT signal transduction pathway and encodes a protein distantly related to the mammalian cytokine receptor family. Genes Dev 2002; 16: 388-98.

17 Dearolf CR. JAKs and STATs in invertebrate model organisms. Cell Mol Life Sci 1999; 55:1578-84.

18 Zeidler MP, Perrimon N, Strutt DI. Polarity determination in the Drosophila eye: a novel role for unpaired and JAK/STAT signaling. Genes Dev 1999; 13:1342-53.

19 Chen X, Oh SW, Zheng Z, et al. Cyclin D-Cdk4 and cyclin ECdk2 regulate the Jak/STAT signal transduction pathway in Drosophila. Dev Cell 2003; 4:179-90.

20 Li J, Xia F, Li WX. Coactivation of STAT and Ras is required for germ cell proliferation and invasive migration in Drosophila. Dev Cell 2003; 5:787-98.

21 Xi R, McGregor JR, Harrison DA. A gradient of JAK pathway activity patterns the anterior-posterior axis of the follicular epithelium. Dev Cell 2003; 4:167-77.

22 Wolff T, Ready DF. Pattern formation in the Drosophila retina. In: Bate M, Martinez Arias A, eds. The Development of Drosophila melanogaster. Cold Spring Harbor Laboratory Press, Cold Spring Harbor, NY, 1993:1277-326.

23 Perrimon N, Mahowald AP. l(1)hopscotch, a larval-pupal zygotic lethal with a specific maternal effect on segmentation in Drosophila. Dev Biol 1986; 118:28-41.

24 Betz A, Lampen N, Martinek S, Young MW, Darnell JE. A Drosophila PIAS homologue negatively regulates stat92E. Proc Natl Acad Sci USA 2001; 98:9563-8.

25 Tsai YC, Sun YH. Long-range effect of $u p d$, a ligand for Jak/ STAT pathway, on cell cycle in Drosophila eye development. Genesis 2004; 39:141-53.

26 Levy DE, Darnell JE. STATS: transcriptional control and bio- logical impact. Nat Rev Mol Cell Biol 2002; 3:651-62.

27 Wolfel T, Hauer M, Schneider J, et al. A p16INK4a-insensitive CDK4 mutant targeted by cytolytic T lymphocytes in a human melanoma. Science 1995; 269:1281-4.

28 Zuo L, Weger J, Yang Q, et al. Germline mutations in the p16INK4a binding domain of CDK4 in familial melanoma. Nat Genet 1996; 12:97-9.

29 Rane SG, Dubus P, Mettus RV, et al. Loss of Cdk4 expression causes insulin-deficient diabetes and Cdk4 activation results in b-islet cell hyperplasia. Nat Genet 1999; 22:44-52.

30 Sherr CJ, Roberts JM. CDK inhibitors: positive and negative regulators of G1-phase progression. Genes Dev 1999; 13:150112.

31 Tulina N, Matunis N. Control of stem cell self-renewal in Drosophila spermatogenesis by JAK-STAT signaling. Science 2001; 294:2546-9.

32 Kiger AA, Jones DL, Schulz C, Rogers MB, Fuller MT. Stem cell self-renewal specified by JAK-STAT activation in response to a support cell cue. Science 2001; 294:2542-5.

33 Brawley C, Matunis E. Regeneration of male germline stem cells by spermatogonial dedifferentiation in vivo. Science $2004 ; 304$ : 1331-4.

34 Fuller MT. Spermatogenesis. In: Bate M, Martinez-Arias A, eds. The Development of Drosophila melanogaster, Cold Spring Harbor Laboratory Press, Cold Spring Harbor, NY, 1993:71147.

35 Spradling A, Drummond-Barbosa D, Kai T. Stem cells find their niche. Nature 2001; 414:98-104.

36 Yamashita YM, Jones DL, Fuller MT. Orientation of asymmetric stem cell division by the APC tumor suppressor and centrosome. Science 2003; 301:1547-50.

37 Kiger AA, White-Cooper H, Fuller MT. Somatic support cells restrict germline stem cell self-renewal and promote differentiation. Nature 2000; 407:750-4.

38 Tran J, Brenner TJ, DiNardo S. Somatic control over the germline stem cell lineage during Drosophila spermatogenesis. Nature 2000; 407:754-7.

39 Matsuda T, Nakamura T, Nakao K, et al. STAT3 activation is sufficient to maintain an undifferentiated state of mouse embryonic stem cells. EMBO J 1999; 18:4261-9.

40 Smith AG. Embryonic stem cells. In: Marshak D, Gardner RL, Gottlieb D, eds. Stem Cells. Cold Spring Harbor Laboratory Press, Cold Spring Harbor, NY, 2001:205-30. 